\title{
Traffic Control Management and Road Safety using Vehicle to Vehicle Data Transmission BASED ON LI-FI TECHNOLOGY
}

\author{
Nilufa Yeasmin ${ }^{1}$, Rianon $\operatorname{Zaman}^{1}$ and Israt Jahan Mouri ${ }^{1}$ \\ ${ }^{1}$ Department of Computer Science and Engineering, Stamford University Bangladesh, \\ Dhaka, Bangladesh
}

\begin{abstract}
An emerging technology $\mathrm{Li}-\mathrm{Fi}$, or light fidelity is a bidirectional and fully networked wireless communications medium which uses light from light-emitting diodes (LEDs) and provides transmission of data through illumination by sending data through a LED light bulb that varies in intensity faster than the human eye can follow. It can provide connectivity within a very large area with more security and with higher data rates and high speed than data that can be transmitted through Wi-Fi. It uses visible light communication or infra-red and near ultraviolet spectrum which works by switching bulbs on and off within nanoseconds. By using Li-Fi technology highly reliable vehicle to vehicle communication is possible by transmitting and receiving data through LED head-lights and tail-lights. In this paper we propose a system that uses Li-Fi enabled LED head-light, tail-light and traffic signal light that can be used for traffic management and road safety by using vehicle to vehicle data transmission The aim of designing this system is to reduce road accidents and managing traffic more accurately.
\end{abstract}

\section{KEYWORDS}

Li-Fi, Visible Light Communication, Arduino, PIC Microcontroller, Light-emitting diodes.

\section{INTRODUCTION}

In today's world Internet is a must for everyone's life. Various activities can be done with the help of Internet. Generally Internet is used for data transmission and people want to transfer data with high speed. For efficient and fast data transmission wireless communication between the devices is used. Wireless communication is needed for every human being. The wireless data is transmitted by the electromagnetic waves.

Now-a-days, Wi-Fi is the main wireless communication medium which uses radio waves and is used in all the areas such as offices, schools, homes, colleges, Universities etc. But the main problems of $\mathrm{Wi}-\mathrm{Fi}$ are scarcity of radio waves, non-stop depletion of bandwidth, RF interferences, expense of bandwidth and the use of repeaters. Other portions of electromagnetic spectrum include X-rays, Gamma rays and Ultraviolet lights but they are not suitable for wireless communication as they are dangerous for human body. Other than the said problems due to extensive usage the radio frequency waves are getting blocked and the capacity is decreasing. In order to overcome this difficulty in future, light fidelity (Li-Fi) technology was invented in 2011 by Dr. Harald Haas which transmits the data through visible light instead of radio waves.

Li-Fi uses LED lamps as the major source of visible light which can be mounted in streets, houses, cars, shops, offices, etc. These LEDs are often referred to as energy savers. So now Li-Fi eliminates the need for repeaters and base stations. Li-Fi also solves the security, hacking of 
networks or tapping of data problem. Li-Fi is a cutting edge technology that offers a relatively easier, cheaper, faster and more secure means of data transfer.

Li-Fi technology has an imperative use in road safety and traffic management. Li-Fi enabled LED head-light, tail-light and traffic signal light can be used for traffic management and road safety using vehicle to vehicle data transmission. In this paper we propose a method to manage traffic and to maintain the safety of a road using LI-Fi technology. We assign each vehicle an unique number against which information is stored in the database. Whenever any car breaks the traffic signal or crosses the maximum speed limit the information against the unique number of the car goes to the central server through Li-Fi. The information from the server will help to take legal actions against the vehicles. Also if any two vehicles cross the minimum distance which is required to prevent accident then a signal is sent to the latter vehicle to slow its speed in order to avoid the accidents.

The paper is arranged as follows: Section 2 provides a brief overview about literature review; Section 3 discusses about the working principle of Li-Fi; Section 4 shows the proposed system design and architecture, and finally, Section 5 draws some conclusions and remarks on the future works.

\section{LITERATURE REVIEW}

LI-FI technology changes the possibility about how we access the internet, videos, audios, emails and many more and the base of it is visible light communication (VLC), which includes for transmitting information is transmitted by using electromagnetic spectrum. The idea of sending information using LED's began during the 1990's and experiments showed that transmitting data wirelessly LEDs can be used.

The idea of Li-Fi was introduced by Harald Haas, the teacher of University of Edinburgh in Scotland and he brought the idea of "Wireless data from every light". Haas and his re-search assistant, Mostafa Afgani, first sent data by using light signals in 2007 [1]. The term Li-Fi was first used by Haas in his TED Global talk on Visible Light Communication. He referred this light signal as D-Light which can be used to produce data rates higher than 10 megabits per second which is much faster than our average broadband connection [2].

Several research works have been carried out on working mechanism of $\mathrm{Li}-\mathrm{Fi}$, on how it provides better bandwidth, efficiency and better availability and security, on how it has immense possibilities, on how Li-Fi differs from other wireless communication mediums [3] [4] [5]. But only a few researches have been carried out on practical implementation of Li-Fi. Researches have been done to show how Li-Fi can be used in Indian Railways, how Li-Fi can be used to park vehicles with intelligence is shown in [6], the procedure of detecting the position of a moving person and updating the location in his device using Li-Fi is illustrated in [7], how Li-Fi in conjunction with PIC microcontroller can be used for traffic management and road safety is shown in [8], how vehicle to vehicle transmission can be used to reduce accidents and the decoding of transmitted data by microcontrollers is shown in [9].

But in our work we proposed the procedure of traffic management, and road safety by using vehicle to vehicle transmission based on $\mathrm{Li}$-Fi but we used Arduino microcontroller to encode and decode data because it is much more effective than PIC microcontroller which is only a chip but the former is a platform. Arduino is much simpler in quick prototyping. 


\section{Working Principle of Li-Fi}

The working principle of light fidelity technology is much simple. The main part of Li-Fi technology is formed with the new generation of high brightness LED which can be switched on and off very fast. If the LED is on binary data 1 is transmitted and if it is off binary 0 is transmitted [10].

A light source or LED bulbs act as transmitter on one end and on the receiver end there is a light sensor or photo detector. When a LED flashes it behaves like a trigger which is then detected by a photo detector. In order to build up a message, the LED is flashed numerous times or an array of LEDs of a few different colors is used to obtain data rates in the range of hundreds of megabits per second [11]. Depending on what the LED bulbs sense, photo detector will give output in the binary format like 0 or 1 [12]. Figure 1 gives a clear picture of working mechanism of Li-Fi.

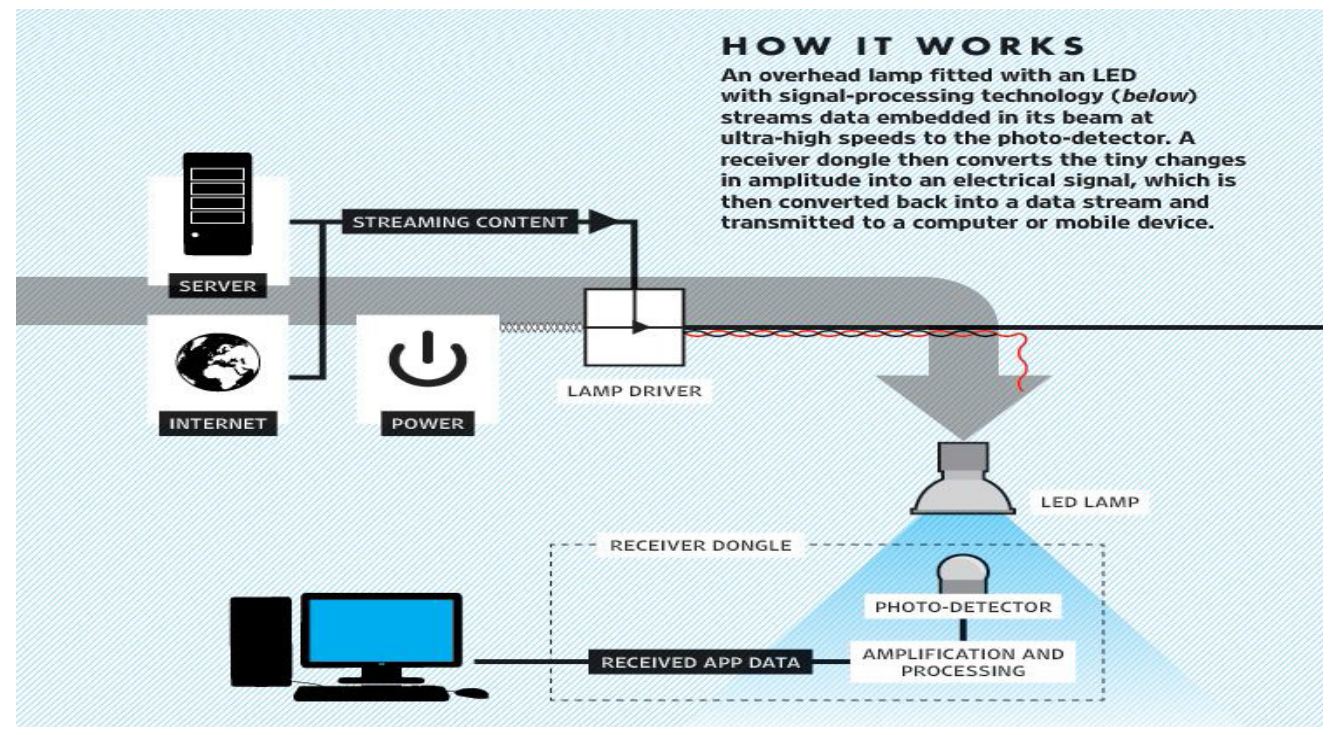

Figure 1.Working mechanism of Li-Fi

\section{Proposed System Design and Overview for Traffic MANAGEMENT AND ROAD SAFETY}

In Li-Fi technology LED lights are the internet access points where visible lights are used for communicating between nodes. $\mathrm{Li}-\mathrm{Fi}$ technology is based on the concept of Visible light communications (VLC) which works by switching bulbs on and off within nanoseconds. Although Li-Fi bulbs would have to be kept on to transmit data, the bulbs could be dimmed to the point that they are not visible to humans and yet still functional. Li-Fi enabled LED head-light, tail-light and traffic signal light can be used for traffic management and road safety using vehicle to vehicle data transmission. The system requires a transmitter and a receiver in each vehicle and the LED head-lights and tail-lights act as transmitter and receiver in both back side and front sides of the vehicle. The traffic signal light also acts as transmitter and receiver. The data to be transmitted is encoded in the head-lights, tail-lights and traffic signal lights. The receiver consists of a data converter and the converted data is then sent to a server or mobile which takes the necessary action [8]. 


\subsection{Traffic Management using Vehicle to Traffic Control Device Data Transmission}

\subsubsection{Managing Traffics}

A very important part of the highway system is Traffic control devices (TCDs) - traffic signs, signals and pavement markings. They provide important information about the roadway to the drivers of vehicles. Traffic signs utilize color, shape, symbols and/or words to convey information. To control traffic, signs using colors are used in the form of four different types of LED lights in roads. The light sequence is: green: safe to cross, yellow or orange: continue to cross only if unable to stop safely, Flashing yellow or orange: cross with caution (often used when lights are out of order or shut down) and red: do not cross [13]. Figure 2 and Figure 3 illustrates this scenario.

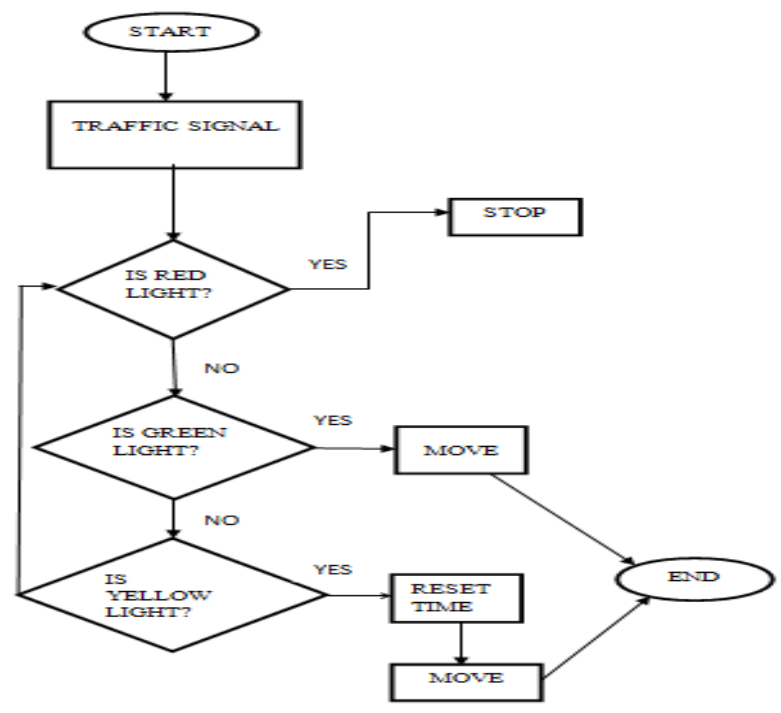

Figure 2. A flow chart defining the meaning of Traffic control signals

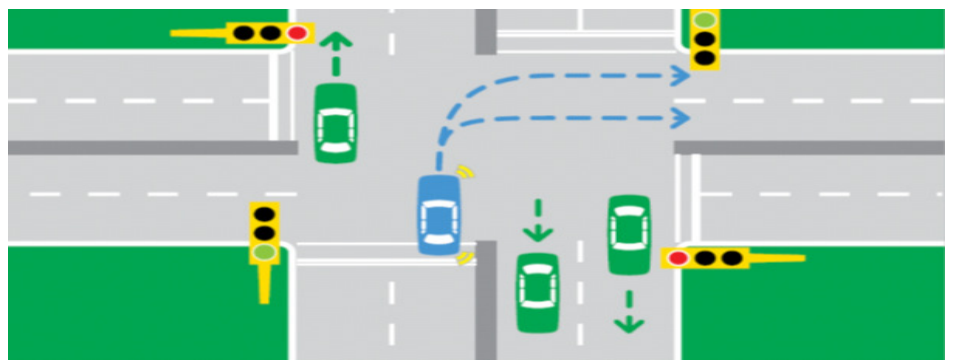

Figure 3. An image defining the meaning of Traffic control signals

All vehicles should be stopped when there is a red signal. But there are some vehicles which do not wish follow this rule. Li-Fi technology can be used to prevent this violence of traffic signal. Every vehicle can be assigned a unique number and information against this unique number must be stored in the central database. The red, green, yellow and orange traffic lights and the head light and tail-lights must be Li-Fi enabled LED which can transmit and receive data. Whenever there is a red traffic signal the server of that spot sends a signal through the red light to all nearby 
vehicles to send that unique code and information about the amount of time they should wait which is received by the head light and tail light of the vehicle. An Arduino microcontroller is used is used to decode the received data from the red light. Then the vehicles must wait that specified amount of time and a timer is generated. If any vehicle starts running before the specified amount of time then that is sensed by the motion sensor attached with the Arduino microcontroller then it sends a signal to the server through head-light or tail-light to red light where complete information about the vehicle is then stored. Traffic police can make use of this information if required and can take necessary action.

\subsubsection{Keeping Speed Limits in Check}

On particular stretches of road speed limits are used to set the maximum speed at which vehicles may legally travel on. Speed limits may be variable or unlimited depending on the place. Speed limits are normally indicated by using a traffic sign [14].

Speed limits can also be kept in check by using Li-Fi technology. The traffic sign where speed limits are indicated a Li-Fi enabled LED can be mounted. All vehicles that pass through that area can be sent a signal from the central computer through the LED on the traffic spot to the headlight which indicates the maximum speed. The data from the head light is then decoded by an Arduino microcontroller connected with that head-light. A LCD display is also attached with the Arduino microcontroller which shows the maximum speed to the driver of the vehicle. Then all vehicles should maintain that specified maximum speed. The speed of the vehicle can be calculated by using Arduino microcontroller with one interrupt pin configured to receive feedback from an optical sensor attached to the motor of the wheel. A photo interrupter can be used to detect rotation by placing a gear with spokes on the drive shaft of the motor so that each spoke will interrupt the beam. This results in a square wave output with a frequency that is proportional to the speed of the motor and the number of spokes on the gear [15]. If the time is recorded at each pulse from the photo interrupter and compared to the time of the previous pulse, the motor rpm can be calculated. Then the microcontroller compares the calculated speed and the specified maximum speed. If the calculated speed is more than the specified speed for that area then that unique code will be send to the server through the head-light of the vehicle and action will be taken against that vehicle.

\subsection{Road Safety using Vehicle to Vehicle Data Transmission}

Communication between vehicles is also possible by using Li-Fi technology. Vehicle to vehicle communication can be used to reduce accidents by calculating distances between vehicles. To calculate distance active ultrasonic sensors are used which generate high-frequency sound waves and evaluate the echo which is received back by the sensor, measuring the time interval between sending the signal and receiving the echo to determine the distance to an object [16]. Each vehicle includes a circuit consisting of ultrasonic sensors, head-lights and tail-lights attached to Arduino microcontroller. Vehicles must maintain a minimum distance from each other. The distances between the vehicles are continuously calculated by the ultrasonic sensor and being compared with the minimum distance. If any two vehicles come closer than the minimum distance then a slowdown command is generated and a signal is sent to the second vehicle to slow down through tail-light to head-light of another vehicle using Li-Fi technology which is then decoded by the Arduino microcontroller. Thus accidents can be reduced.

Figure 4 gives a summarize picture of the above discussion. 


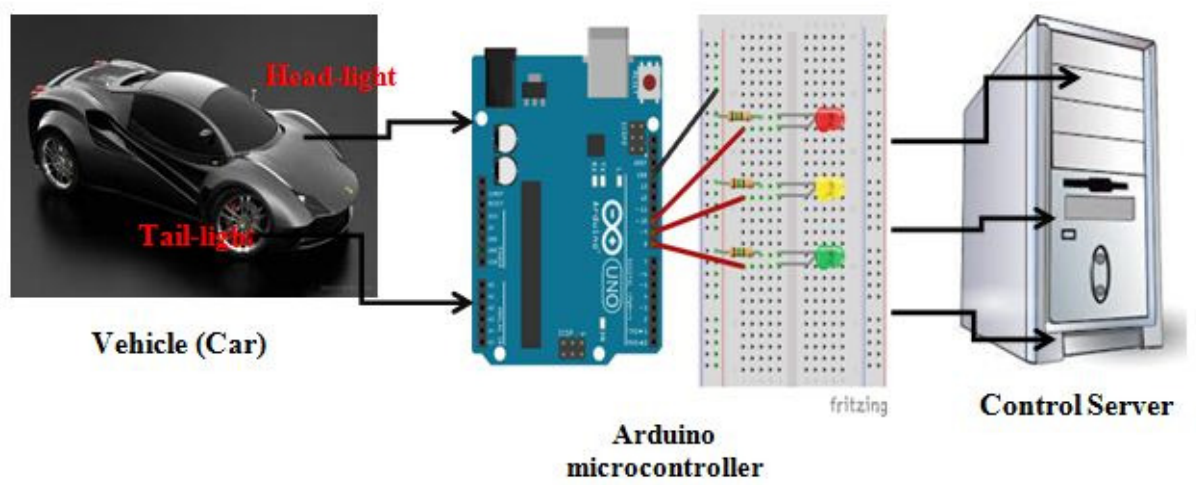

Figure 4. An image defining the Vehicle to Data Transmission using Li-Fi technology .

\section{CONCLUSIONS}

Li-Fi technology has a massive use in traffic management and in establishing safe movements of vehicles on the road. But to implement it commercially each and every vehicle has to include a LED-based traffic head-light, tail-light and Arduino microcontroller. But the fact is, Li-Fi technology is more costly than any other existing one. If we can somehow minimize the cost to some extent, then the there is no need of any traffic police on the road. Again we will not face any unnatural death on the road due to recklessness of the drivers. So it is high time we implement this fabulous technology to step forward to a digital world.

\section{ACKNOWLEDGEMENTS}

The authors would like to thank everyone, just everyone!

\section{REFERENCES}

[1] "Li-Fi", Wikipedia, 2016. [Online]. Available: https://en.wikipedia.org/wiki/Li-Fi. [Accessed: 25Jun- 2016].

[2] "Forget WiFi, It's LiFi: Internet Through Lightbulbs", GOOD Magazine, 2011. [Online]. Available:https://www.good.is/articles/forget-wifi-it-s-lifi-internet-through-lightbulbs. [Accessed: 25Jun- 2016].

[3] A. Abdulhussain Shuriji, "An Extensive Comparison of the Next Generation of Wireless Communication Technology: Light-Fidelity (Li-Fi) Versus Wireless-Fidelity (Wi-Fi)", GSTF Journal on Media \& Communications, vol. 2, no. 1, 2014.

[4] N.,"LI-FI (LIGHT FIDELITY) - THE CHANGING SCENARIO OF WIRELESS COMMUNICATION", International Journal of Research in Engineering and Technology, vol. 04, no. 03, pp. 435-438, 2015.

[5] "A Comparison between Li-Fi, Wi-Fi, and Ethernet Standards", IJSR, vol. 4, no. 12, pp. 1-4, 2015.

[6] N. Kim, C. Jing, B. Zhou and Y. Kim, "Smart Parking Information System Exploiting Visible Light Communication", IJSH, vol. 8, no. 1, pp. 251-260, 2014.

[7] S. "LIGHT FIDELITY FOR POSITION DETECTION", International Journal of Research in Engineering and Technology, vol. 04, no. 01, pp. 1-4, 2015.

[8] Kapre, Mrs Kshitija Suhas. "Road Traffic Management and Safety Using Li Fi Technology." Road Traffic Management 2.12 (2015).

[9] Patel, Mr Jagdish A., et al. "Li-Fi Technology-Vehicle to Vehical Data Transmission."

[10] Adwani, Anshu, and Smita Nagtode. "LI-FI: INFORMATION TRANSFERRING THROUGH LED'S."

[11] Sharma, Rahul R., and Akshay Sanganal. "Li-Fi Technology: Transmission of data through light." International Journal of Computer Technology and Applications 5.1 (2014): 150.

[12] S. Gupta, S. Gupta and S. Pandey, "Study \& Analysis of Role of Li-fi in Future", IJSH, vol. 10, no. 2, pp. 213-218, 2016. 
[13] "Traffic light", Wikipedia, 2016. [Online]. Available: https://en.wikipedia.org/wiki/Traffic_light. [Accessed: 25- Jun- 2016].

[14] "Speed limit", Wikipedia, 2016. [Online]. Available: https://en.wikipedia.org/wiki/Speed_limit. [Accessed: 25- Jun- 2016].

[15] Using Arduino Microcontrollers to Sense DC Motor Speed and Position, 1st ed. 2016.

[16] "Ultrasonic transducer", Wikipedia, 2016. [Online]. Available: https://en.wikipedia.org/wiki/Ultrasonic_transducer. [Accessed: 25- Jun- 2016].

\section{Authors}

Nilufa Yeasmin is a lecturer in the Department of Computer Science and Engineering, Stamford University Bangladesh, Dhaka, Bangladesh. She received her B.Sc in Computer Science and Engineering from Stamford University Bangladesh (SUB), Dhaka, Bangladesh in 2014. She passed her Higher Secondary Certificate (H.S.C) and Secondary School Certificate (S.S.C) from Alimuddin Degree College, Lalmonirhat, Bangladesh in 2009 and Shah Goribullah Girls High School, Lalmonirhat, Bangladesh respectively in 2007. Her research interest includes Wireless Data Mining, Sensor Network and Software Engineering etc.

Rianon Zaman is a lecturer in the Department of Computer Science and Engineering, Stamford University Bangladesh, Dhaka, Bangladesh. She received her B.Sc in Computer Science and Engineering from Khulna University of Engineering and Technology, Bangladesh in 2015. She passed her Higher Secondary Certificate (H.S.C) and Secondary School Certificate (S.S.C) form Holy Cross College, Dhaka, Bangladesh in 2010 and Holy Cross Girls' High School, Dhaka, Bangladesh in 2008. Her research interest includes Bioinformatics, Data Mining, Machine Learning etc.

Israt Jahan Mouri is a lecturer in the Department of Computer Science and Engineering, Stamford University Bangladesh, Dhaka, Bangladesh. She received her B.Sc in Computer Science and Engineering from Military Institute of Science \& Technology (MIST), Dhaka, Bangladesh in 2014. She passed her Higher Secondary Certificate (H.S.C) and Secondary School Certificate (S.S.C) from Rajuk Uttara Model College, Dhaka, Bangladesh in 2009 and The Buds Residential Model College, Sylhet, Bangladesh respectively in 2007. Her research interest includes Wireless Ad Hoc Networks, Social Network Security and Android Mobile Security etc.
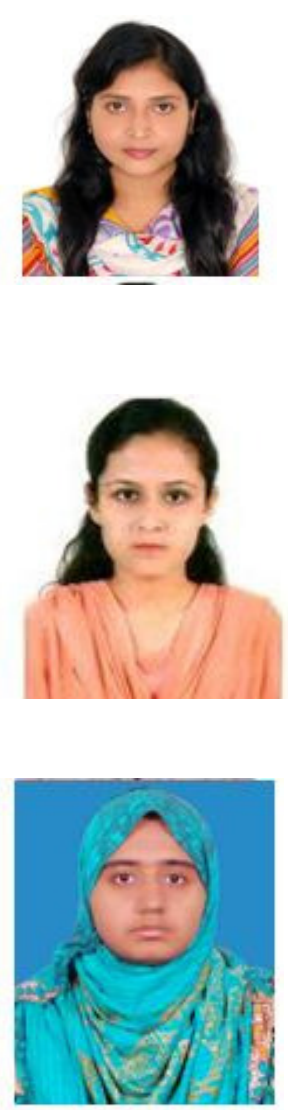